\title{
Does the maxillary sinus have a triggering role in nasal nitric oxide synthesis?*
}

\author{
Oğuz Güçlü', Ahmet Uludağ², Alper Akçalı, Kazım Tekin', Halil Erdoğan', \\ Fatma Sılan², Fevzi Sefa Dereköy \\ Department of Otorhinolaryngology, Canakkale Onsekiz Mart University Faculty of Medicine, Çanakkale, Turkey \\ Department of Medical Genetic, Canakkale Onsekiz Mart University Faculty of Medicine, Çanakkale, Turkey \\ Department of Microbiology, Canakkale Onsekiz Mart University Faculty of Medicine, Çanakkale, Turkey
}

Rhinology 50: 402-407, 2012

DOI:10.4193/Rhino12.081

*Received for publication:

May 19, 2012

Accepted: August 20, 2012

\section{SUMMARY}

Objectives: We investigated whether the maxillary sinus plays a stimulatory role in nasal nitric oxide (NO) synthesis. Research on sinusitis and nasal polyps has found low NO levels in exhaled air and linked this to obstruction of the ostium. However, the major source of NO in exhaled air is thought to be the nasal mucosa. In this study, Streptococcus pneumoniae was applied to the maxillary sinus to investigate changes in NO synthesis of the nasal mucosa.

Methods: An experimental study was performed with New Zealand white rabbits. Three groups, pneumococcus, control and sham, were created. The maxillary sinus of the pneumococcal group was exposed to Streptococcus pneumoniae suspension. Before and after the exposure, bilateral biopsy specimens were taken from the inferior turbinate. Specimens were examined by RT-PCR for expressions of endothelial nitric oxide synthase (e-NOS) and inducible nitric oxide synthase (i-NOS). Physiological saline solution was administered to the maxillary sinus in the control group and biopsies were obtained. The sham group underwent only biopsy.

Results: A significant increase in i-NOS expression of tissue samples from the pneumococcal group on the same and opposite sides were detected. There was no increase in e-NOS expression in this group. The control and sham groups had no significant change in i-NOS or e-NOS expression.

Conclusion: In the acute period after the maxillary sinus is exposed to a pathogen, i-NOS expression increases in the nasal mucosa, but endothelial NOS expression is not affected. Consequently, a combined response in the maxillary sinus and the nasal mucosa for nitric oxide synthesis is shown in the present study.

Key words: maxillary sinus, nitric oxide, NOS, inducible NOS, expression

\section{Introduction}

Nitric oxide (NO) is a mediator that plays a role in numerous biological activities such as circulation, platelet functions, immunity and neurotransmission. It is synthesized from L-arginine, a semi-essential amino acid, through nitric oxide synthase (NOS). NOS has three isoforms: endothelial NOS (eNOS), neuronal NOS (nNOS), and inducible NOS (iNOS). NO is found in its gas form in breathing air and its major source is the upper respiratory airway mucosa. It is thought to function as a part of the defense system against infections ${ }^{(1)}$.

NO has pro-inflammatory and anti-inflammatory effects as a mediator in nasal physiology (2). $\mathrm{NO}$ amount is significantly increased in case of inflammation (i.e. allergic rhinitis). Lundberg demonstrated that the maxillary sinus was the main source of NO ${ }^{(3)}$. However, Haight et al. have showed that $12 \%$ of NO in 
nasal breathing air originates from maxillary sinus ${ }^{(4)}$. Thus, nasal epithelium has a significant role in NO production and upper respiratory mucosa is the source of most of the NO in exhaled air $(3,5)$. The amount of $\mathrm{NO}$ in exhaled air is reduced during inflammation such as chronic sinusitis and nasal polyposis in which the osteomeatal complex is blocked ${ }^{(1,2)}$. NO produced in the maxillary sinus cannot be transferred into nasal breathing air when the ostium is closed due to inflammation. However, this also leads to a contradiction that the NO level, which is supposed to be increased during inflammation, does not change and the contribution of paranasal sinuses to the amount of NO in exhaled air is low even if the osteomeatal complex is blocked. This suggests a completely different question whether inflammation in maxillary sinus induces an inflammatory response in the nasal mucosa, which is at the outside of the maxillary sinus. The aim of the present study was to investigate if inflammation in maxillary sinus had a triggering role or common response in inducing an inflammatory response in the nasal mucosa.

\section{Materials and method}

Animal study

The present study was conducted in the Department of Otolaryngology in Canakkale Onsekiz Mart University. The study was approved by the local Ethics Committee. Twenty-one 6-month old New Zealand white rabbits were included and taken care of under optimum conditions in the animal laboratory. Three groups were formed as pneumococcus, sham and control. A sufficient number of Streptococcus pneumoniae (ATCC 49619) colonies grown on $5 \%$ sheep blood agar plates were suspended in sterile saline and adjusted to a $0.5 \mathrm{McFarland}$ turbidity standard using the DensiChek densitometer (bioMerieux, France). Anesthesia was induced by intramuscular ketamine $(35 \mathrm{mg} / \mathrm{kg})$ and xylazine $(5 \mathrm{mg} / \mathrm{kg}$ ) injection.

Right maxillary sinus was chosen as the intervention site. Maxillary sinus was accessed by a syringe from approximately $1 \mathrm{~cm}$ anterior and inferior of the eye. During this procedure, injection depth was determined based on the level of puncture of the thin bone lamella located in the anterior wall of the maxillary sinus and $0.1 \mathrm{ml}$ of bacterial suspension was injected into the sinus. Three nasal mucosa biopsies were obtained from the inferior nasal concha using a micro-cup forceps before and one and five minutes after the procedure. The procedure was performed for both right and left nasal cavity. Tissue biopsies were performed from anterior, middle and posterior regions of the concha and stored at $-80^{\circ} \mathrm{C}$.

In the control group, $0.1 \mathrm{ml}$ of $0.9 \% \mathrm{NaCl}$ was injected into the maxillary sinus and tissue samples were obtained. In the sham group, tissue samples were obtained without any intervention in maxillary sinus.
NOS expression analysis

Rabbit nasal mucosa tissues were used for genomic RNA isolation and cDNA synthesis. Total genomic RNA was extracted from rabbit mucosa from each sample by the High Pure RNA tissue kit (Roche Diagnostics GmbH, Germany) and cDNA by the Transcriptor first strand cDNA synthesis kit (Roche Diagnostics). The NOS2 and NOS3 expressions were detected using LightCycler TaqMan Master kit by LightCycler 2.0 real time PCR system (Roche Diagnostics). For the expression analysis, $4 \mu \mathrm{l}$ of LightCycler TaqMan Master mix, $1.4 \mu \mathrm{l}$ of PCR-grade water, $0.2 \mu \mathrm{l}$ of forward primer, $0.2 \mu \mathrm{l}$ of reverse primer, $0.2 \mu \mathrm{l}$ of probe and $5 \mu \mathrm{l}$ of cDNA template were used. The hypoxanthine-guanine phosphoribosyltransferase (HPRT) gene was used as a reference gene in the detection of NOS2(iNOS) and NOS3(eNOS) expressions in the tissues.

The LightCycler 2.0 (Roche Diagnostics) system software program was used for the detection of gene expressions and relative quantification-monocolour was performed in the detection of expression levels.

\section{Statistical analysis}

Data analysis was performed using Statistical Package for the Social Sciences (SPSS) version 16.0 for windows. The iNOS and eNOS levels were calculated as mean \pm standard deviation in the study groups. Friedman test was used for the intragroup comparisons and Kruskal-Wallis test was used for the intergroup comparisons. $p$ values $<0.05$ were considered statistically significant.

\section{Results}

According to our results, statistically significant increase in iNOS expression was noted in the right side biopsies, one and five minutes after the procedure in the pneumococcus group $(\mathrm{p}=$ 0.001). However, no increase in eNOS expression was observed. There was no significant change in iNOS or eNOS expression levels in the control and sham groups (Table 1 and 2).

Statistically significant increase in iNOS expression was also noted in the left side biopsies after the procedure in the pneumococcus group ( $p=0.002$ ); however, there was no significant change in eNOS expression. Moreover, there was no significant change in both gene expressions in the control and sham groups (Table 3 and 4).

\section{Discussion}

Nitric oxide, a free radical gas, is produced by various cells throughout the body and serves to regulate a vast number of physiological and pathophysiological processes including blood flow, neurotransmission, host defense, platelet function and inflammation ${ }^{(1-3)}$. NO is synthesized by NOS, which has three isoforms: eNOS, nNOS and iNOS ${ }^{(1)}$. In the literature, the roles of 
Table 1. Inducible NOS (i-NOS) expression values of the right nasal mucosa biopsy samples before the procedure, and in the first and fifth minute after.

\begin{tabular}{|c|c|c|c|c|}
\hline Right nasal cavity & $\begin{array}{l}\text { i-NOS before procedure } \\
\qquad\left(10^{-4}\right)\end{array}$ & $\begin{array}{c}\text { i-NOS } \\
\text { 1st minute }\left(10^{-4}\right)\end{array}$ & $\begin{array}{c}\text { i-NOS } \\
\text { 5th minute }\left(10^{-4}\right)\end{array}$ & $p$ value \\
\hline Pneumococcus & $2,7 \pm 1,25$ & $146,41 \pm 96,55$ & $2375,82 \pm 2057,90$ & $0,001^{*}$ \\
\hline Sham & $1,44 \pm 0,59$ & $0,78 \pm 0,31$ & $4,02 \pm 2,02$ & 0,368 \\
\hline Control & $1,26 \pm 0,44$ & $1,48 \pm 0,90$ & $17,9 \pm 13,92$ & 0,066 \\
\hline$p$ value & 0,58 & $0,009^{*}$ & $0,003^{*}$ & \\
\hline
\end{tabular}

*: statistically significantt

Table 2. Endothelial NOS (e-NOS) expression values of the right nasal mucosa biopsy samples before the procedure, and in the first and fifth minute after.

\begin{tabular}{|c|c|c|c|c|}
\hline Right nasal cavity & $\begin{array}{c}\text { e-NOS } \\
\text { before procedure }\end{array}$ & $\begin{array}{c}\text { e-NOS } \\
\text { 1st minute }\end{array}$ & $\begin{array}{c}\text { e-NOS } \\
\text { 5th minute }\end{array}$ & p value \\
\hline Pneumococcus & $0,25 \pm 0,06$ & $0,3 \pm 0,08$ & $0,33 \pm 0,13$ & 0,867 \\
\hline Sham & $0,20 \pm 0,04$ & $0,1 \pm 0,03$ & $0,25 \pm 0,09$ & 0,097 \\
\hline Control & $0,23 \pm 0,06$ & $0,28 \pm 0,08$ & $0,23 \pm 0,04$ & 0,651 \\
\hline$p$ value & 0,791 & 0,61 & 0,797 & \\
\hline
\end{tabular}

NO in the upper airways have been reported as protection from infections as the part of host defense, mediators of pro-inflammatory and anti-inflammatory effects and regulators of mucociliary clearance ${ }^{(2)}$. Inducible NOS is expressed in white blood cells, epithelial cells and other cells in response to proinflammatory cytokines and bacterial components. During inflammation, iNOS is upregulated and NO concentration increases. High NO concentration and its products are cytotoxic to viruses, bacteria, tumor cells and possibly even host cells. Thus, their benefit or harm to the host during inflammation is still an issue of debate (3).

In 1991, Gustafsson et al., evaluated the presence NO in exhaled breath of the experimental animals ${ }^{(6)}$. Two years later, Alving et al. found higher NO levels in nasal breath compared to those in orally exhaled air, which suggested a contribution from the upper airways ${ }^{(7)}$. However, in their study conducted on cases with tracheostomy, Lundberg et al. reported higher NO levels in nasal exhalation, intermediate levels in oral breath, and lower NO levels in air exhaled through the tracheostomy ${ }^{(3)}$. Analyses on the amount of NO by aspiration of maxillary sinus have revealed that maxillary sinus is the major area containing NO in the sino-nasal region ${ }^{(3)}$. On the contrary, in their study, Haight et al. suggested that the main source of NO in exhaled air was nasal mucosa. They found that blocking the osteomeatal complex and the spheno-ethmoid recess resulted in only $12 \%$ decrease in the overall nasal NO concentration ${ }^{(4)}$. However, further studies are still needed to identify the main source of NO.

NO can be measured directly or indirectly. With the indirect method, NOS expression and products of NO metabolism are analyzed; whereas, with the direct method NO is measured by chemiluminescence, in which detection is based on the photochemical reaction between $\mathrm{NO}$ and ozone ${ }^{(1)}$. In the present study, NOS expression was evaluated in tissue samples by PCR analysis of RNA levels.

In many studies, the association between NO and inflammatory conditions such as allergy, nasal polyposis or asthma has been evaluated ${ }^{(8)}$. Li et al. reported an increase in iNOS expression in experimental animals that come into contact with an allergen, while there was no change in eNOS and nNOS expression ${ }^{(9)}$. Yüksel et al. and Chiba et al. also found similar results ${ }^{(10,11)}$. In a study, Takeno et al. reported that higher levels of NO was produced through the concomitant expression of different NOS isoforms (NOS2 and NOS3) in the nasal epithelial cells of the patients with 
Table 3. Inducible NOS (i-NOS) expression values of the left nasal mucosa biopsy samples before the procedure, and in the first and fifth minute after.

\begin{tabular}{|c|c|c|c|c|}
\hline Left nasal cavity & $\begin{array}{l}\text { i-NOS before procedure } \\
\qquad\left(\times 10^{-4}\right)\end{array}$ & $\begin{array}{c}\text { i-NOS } \\
\text { 1st minute }\left(\times 10^{-4}\right)\end{array}$ & $\begin{array}{c}\text { i-NOS } \\
\text { 5th minute }\left(\times 10^{-4}\right)\end{array}$ & p value \\
\hline Pneumococcus & $14,36 \pm 9,44$ & $392,09 \pm 368,03$ & $288,21 \pm 240,73$ & $0,002^{*}$ \\
\hline Sham & $0,58 \pm 0,22$ & $0,61 \pm 0,29$ & $3,14 \pm 2,17$ & 0,895 \\
\hline Control & $1,07 \pm 0,43$ & $2,04 \pm 0,96$ & $2,25 \pm 1,38$ & 0,867 \\
\hline$p$ value & 0,106 & $0,007^{*}$ & $0,007^{*}$ & \\
\hline
\end{tabular}

*: Statistically significant

Table 4. Endotelial NOS (e-NOS) expression values of the left nasal mucosa biopsy samples before the procedure, and in the first and fifth minute after.

\begin{tabular}{|c|c|c|c|c|}
\hline Left nasal cavity & $\begin{array}{c}\text { e-NOS } \\
\text { before procedure }\end{array}$ & $\begin{array}{c}\text { e-NOS } \\
\text { 1st minute }\end{array}$ & $\begin{array}{c}\text { e-NOS } \\
\text { 5th minute }\end{array}$ & p value \\
\hline Pneumococcus & $0,12 \pm 0,02$ & $0,07 \pm 0,01$ & $0,12 \pm 0,04$ & 0,191 \\
\hline Sham & $0,16 \pm 0,05$ & $0,14 \pm 0,03$ & $0,13 \pm 0,07$ & 0,156 \\
\hline Control & $0,25 \pm 0,07$ & $0,25 \pm 0,07$ & $0,33 \pm 0,09$ & 0,368 \\
\hline $\mathrm{p}$ value & 0,248 & $0,044^{*}$ & 0,106 & \\
\hline
\end{tabular}

allergic rhinitis ${ }^{(12)}$. Furthermore, in recent studies, high levels of iNOS expression and activity have been demonstrated in nasal polyp tissue samples ${ }^{(13,14)}$.

In various studies in the literature, pathogenic microorganisms have been shown to increase the epithelial expression of iNOS. In 1998, Hess et al. revealed an increase in iNOS expression in the nasal mucosa exposed to bacterial lipopolysaccharides ${ }^{(15)}$. In their study Dowling et al. used Pseudomonas aeruginosa to infect the nasal epithelial cell culture and reported an increase in iNOS expression ${ }^{(16)}$. Furthermore, Stark et al. demonstrated induced NO production and increased iNOS activity against respiratory syncytial virus (RSV) ${ }^{(17)}$. Likewise, a number of respiratory viruses including adenovirus, rhinovirus, and influenza virus have been found to induce the production of $\mathrm{NO}^{(17)}$. In a study in which the tissue samples from inferior turbinate mucosa have been examined in chronic rhino-sinusitis patients, an increase in NO expression has been observed ${ }^{(18)}$. On the contrary, several studies have reported a decrease in the concentration of NO in nasal exhaled breath. In another study, reduced NO levels in acute maxillary sinusitis have been reported to be improved after antibiotic therapy ${ }^{(19)}$. Moreover, lower NO concentration levels in cases with chronic sinonasal infection compared to those in the control cases have been reported in several studies ${ }^{(20,21)}$. Some authors have also suggested that blocking of osteomeatal complex due to sinusitis lead to decrease in nasal NO concentration. Alobid et al. noted an increase nasal NO in patients with severe nasal polyposis and treated with steroids. They suggested that steroid treatments improve increase NO by opening osteomeatal complex ${ }^{(22)}$.

Obstruction of osteomeatal complex may lead to impairment of the communication between the nasal cavity and maxillary sinus and, thus, reduces the nasal NO concentration in exhaled air. On the other hand, pathologic microorganisms or infection leads to an increase in the NOS expression in sino-nasal mucosa. Accordingly, the results of studies evaluating the effects of sinus infections on the concentration of NO in nasal breathing air and NOS tissue expression level are controversial. Naturally, obstruction of the ostium is expected to affect the NO concentration in the exhaled air in this proportion. Presence of pathologic microorganisms also leads to upregulation of NOS expression and an increase in the NO concentration.

To our knowledge, there have been no studies investigating the presence of infected material within the maxillary sinus and 
whether it affects the NOS expression in the nasal mucosa or not. In the present study, we investigated the acute changes in NOS expression in the nasal mucosa following injection of bacterial suspension into the maxillary sinus. The levels of iNOS and eNOS expressions in the tissue samples obtained from inferior turbinate following pneumococcus injection into maxillary sinus were evaluated. Bacterial suspension was injected into the right maxillary sinus and samples were obtained from both right and left sides. There was a significant increase in iNOS expression in the tissue samples obtained from both nasal cavities in the pneumococcus group. However, we did not observe an increase in the eNOS expression in these tissue samples. Our results suggested that there was an acute increase in NO synthesis in the nasal mucosa in response to the presence of infected material within the maxillary sinus. In addition, there was a common response against infected material in the maxillary sinus and nasal mucosa. It might be proposed that the infective material may itself reach the nasal cavity and induce this response. However, we observed a similar increase in the iNOS expression in the contralateral side tissue samples as well.

Considering that maxillary sinus and nasal mucosa develop a common response and most of the NO in breathing air originates from the nasal mucosa, question arises as to why NO concentrations are noted to be low in chronic sinusitis patients? Firstly, paranasal sinuses may be contributing to nasal NO concentration more than it was noted in the Haight et al. study and this reduction may be due to ostium obstruction. Secondly, there may be a reduction in NOS expression in the presence of chronic sinus infection. Several cytokines such as IL-4, IL-6 and transforming growth factor have been detected in the sinus mucosa in the patients with chronic sinusitis and these cytokines have been shown, both in vitro and in vivo, to decrease NOS expression ${ }^{(1)}$. In the present study, there was an increase in the NOS expression during initial contact with the pathogen, but there might have also been reduction at some point during the sinusitis process. Expression in nasal mucosa might have also been reduced due to the common response. Thirdly, there might be an interruption between the mucosa and lumen, possibly due to excessive mucoid secretions. It has been demonstrated in a study evaluating NO concentration inside the maxillary sinus lumen air that NO level was normal in repeated aspirations and that there was a constant production ${ }^{(23)}$. Thus, there might be a mechanism in the mucosa detecting the reduction in NO concentration within the lumen and there might be a reduction in NO level due to impairment in the relationship between mucosa and lumen. However, further studies are needed to elucidate these assumptions.

Our study has some limitations as this study also showed a response against S. pneumonia, which is a common pathogen of acute rinosinusitis rather than chronic rinosinusitis. Our findings including acute changes in iNOS in an acute bacterial sinusitis model may have little relevance to chronic sinusitis that's been going on for months and may be more inflammatory rather than bacterial in nature, especially if it is a polypoid disease. Furthermore, we investigated a common response in maxillary sinus and nasal mucosa to the pathogen, not an exact sinusitis model. Of course, results in animals cannot always be extrapolated to man. Additionally, our study is designed with a lower number of cases. However, we found an increase in NOS expression in first and fifth minute biopsy samples. It can be thought that this is a rapid inflammatory response like an allergic reaction, but further studies are needed to define the involved mechanisms.

\section{Conclusion}

In the present study, we found that there was an acute increase in the iNOS expression in the nasal mucosa in response to the injection of a pathogenic microorganism (Streptococcus pneumoniae into the maxillary sinus. A common response in terms of NO synthesis in the maxillary sinus and nasal mucosa was noted. It was also noted that eNOS was not affected. NO is an important molecule in the upper respiratory airway defense mechanism; however, there have been studies showing that its production is decreased in the presence of active sinus infection. Further studies are needed to elucidate the exact mechanism.

\section{Conflict of interest}

All authors declared to have no financial or personal relationships with other people or organizations that could influence (bias) our work.

\section{Acknowledgements}

All authors thank Erkan Melih Sahin for the statistical analyses.

\section{References}

1. Maniscalco M, Sofia M, Pelaia G. Nitric oxide in upper airways inflammatory diseases. Inflamm Res. 2007; 56: 58-69.

2. Oh CK, Miman MC, Duncavage JA. Current value of nasal nitric oxide measurement in rhinology. Curr Opin Otolaryngol Head Neck Surg. 2004; 12: 30-33.

3. Lundberg JO. Nitric oxide and the paranasal sinuses. Anat Rec (Hoboken). 2008; 291
1479-1484.

4. Haight JS, Djupesland PG, Qjan W, et al. Does nasal nitric oxide come from the sinuses? J Otolaryngol. 1999; 28: 197-204.

5. Lundberg JO, Weitzberg E. Nasal nitric oxide in man. Thorax. 1999; 54: 947-952.

6. Gustafsson LE, Leone AM, Persson MG, Wiklund NP, Moncada S. Endogenous nitric oxide is present in the exhaled air of rabbits, guinea pigs and humans. Biochem Biophys
Res Commun. 1991; 181: 852-857.

7. Alving $K$, Weitzberg E, Lundberg JM Increased amount of nitric oxide in exhaled air of asthmatics. Eur Respir J. 1993; 6: 13681370 .

8. Scadding G, Scadding GK. Update on the use of nitric oxide as a noninvasive measure of airways inflammation. Rhinology. 2009; 47: 115-120

9. Li Y, Wang Y, Zhang Q. Expression of cell 
adhesion molecule and nitric oxide synthase in nasal mucosa in allergic rhinitis. Lin Chuang Er Bi Yan Hou Ke Za Zhi. 2006; 20: 315-318.

10. Yuksel H, Kirmaz C, Yilmaz O, et al. Nasal mucosal expression of nitric oxide synthases in patients with allergic rhinitis and its relation to asthma. Ann Allergy Asthma Immunol. 2008; 100: 12-16.

11. Chiba Y, Matsuo K, Sakai H, Abe K, Misawa $M$. Increased expression of inducible nitric oxide synthase in nasal mucosae of guinea pigs with induced allergic rhinitis. Am J Rhinol. 2006; 20: 336-341.

12. Takeno S, Osada R, Furukido K, Chen JH, Yajin $\mathrm{K}$. Increased nitric oxide production in nasal epithelial cells from allergic patients-RT-PCR analysis and direct imaging by a fluorescence indicator: DAF-2 DA. Clin Exp Allergy. 2001; 31: 881-888.

13. Ozcan C, Apa DD, Pata YS, Görür K, Akbaş $Y$. Expression of inducible nitric oxide synthase in antrochoanal polyps. Int J Pediatr Otorhinolaryngol. 2003; 67: 383-838.

14. Ramis I, Lorente J, Roselló-Catafau J, Quesada P, Gelpí E, Bulbena O. Differential activity of nitric oxide synthase in human nasal mucosa and polyps. Eur Respir J. 1996; 9: 202-206.

15. Hess A, Bloch W, Rocker J, Addicks K, Stennert $E$, Michel $O$. In vitro expression of inducible nitric oxide synthase in the nasal mucosa of guinea pigs after incubation with lipopolysaccharides or cytokines. Eur Arch Otorhinolaryngol. 1998; 255: 448-453.

16. Dowling RB, Newton R, Robichaud A, Cole PJ, Barnes PJ, Wilson R. Effect of inhibition of nitric oxide synthase on Pseudomonas aeruginosa infection of respiratory mucosa in vitro. Am J Respir Cell Mol Biol. 1998; 19: 950-958.

17. Stark JM, Khan AM, Chiappetta $\mathrm{CL}$, Xue $\mathrm{H}$, Alcorn JL, Colasurdo GN. Immune and functional role of nitric oxide in a mouse model of respiratory syncytial virus infection. J Infect Dis. 2005; 191: 387-395.

18. Furukawa K, Harrison DG, Saleh D, Shennib $\mathrm{H}$, Chagnon FP, Giaid A. Expression of nitric oxide synthase in the human nasal mucosa. Am J Respir Crit Care Med. 1996; 153: 847850.

19. Baraldi E, Azzolin NM, Biban P, Zacchello F. Effect of antibiotic therapy on nasal nitric oxide concentration in children with acute sinusitis. Am J Respir Crit Care Med. 1997; 155: 1680-1683.

20. Deja M, Busch T, Bachmann S, et al. Reduced nitric oxide in sinus epithelium of patients with radiologic maxillary sinusitis and sepsis. Am J Respir Crit Care Med. 2003; 168: 281-286.

21. Degano B, Génestal M, Serrano E, Rami J,
Arnal JF. Effect of treatment on maxillary sinus and nasal nitric oxide concentrations in patients with nosocomial maxillary sinusitis. Chest. 2005; 128: 1699-1705.

22. Alobid I, Benitez P, Valero A, Munoz R, Langdon C, Mullol J. Oral and intranasal steroid treatments improve nasal patency and paradoxically increase nasal nitric oxide in patients with severe nasal polyposis. Rhinology. 2012; 50: 171-177.

23. Lundberg JO, Farkas-Szallasi T, Weitzberg E, et al. High nitric oxide production in human paranasal sinuses. Nat Med. 1995; 1: 370373.

Asist. Prof. Dr. Oğuz Güçlü Department of Otorhinolaryngology Çanakkale Onsekiz Mart University Faculty of Medicine 17100 Çanakkale Turkey

Tel: $+90-542-2707866$

Fax: +90-286-218 0393

E-mail: droguzguclu@hotmail.com

ADVERTISEMENT

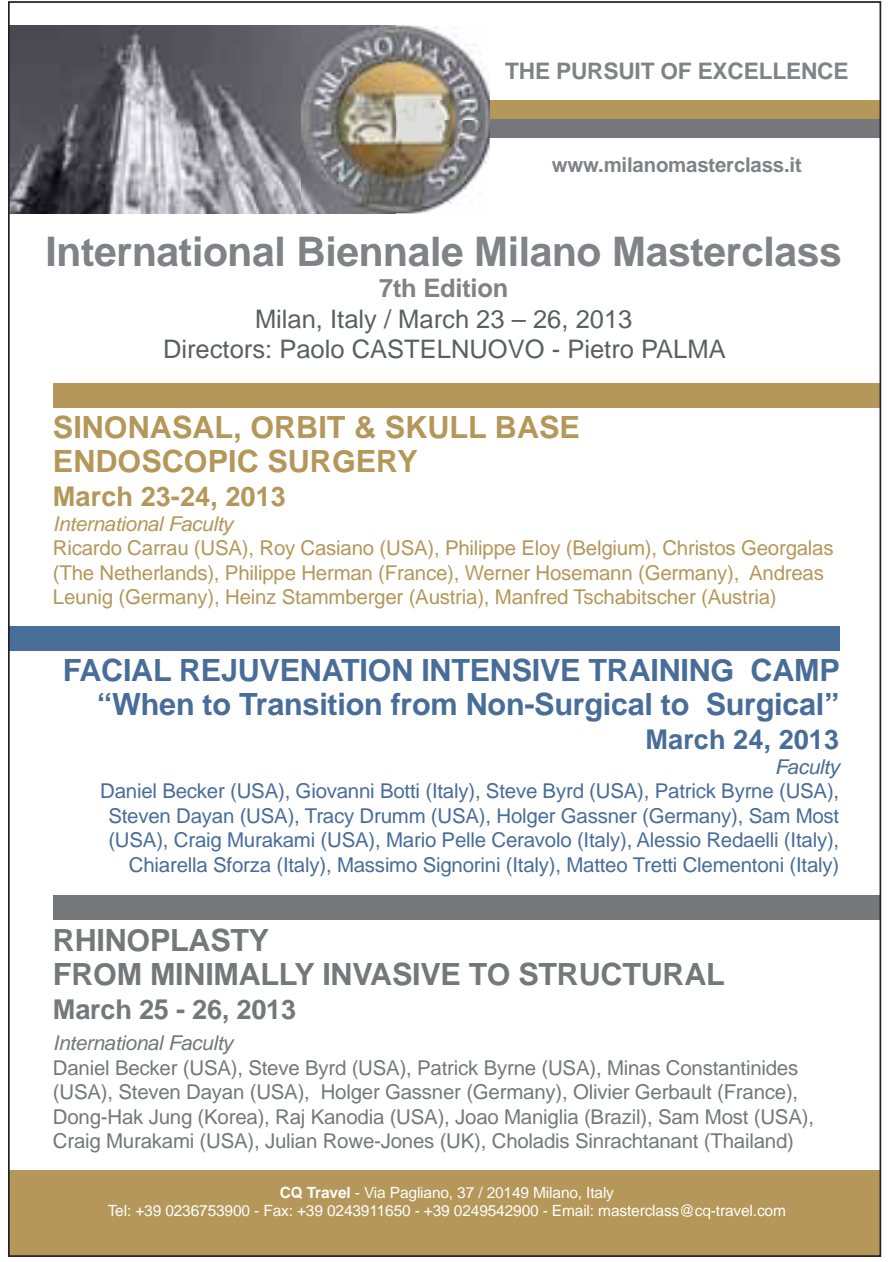

